\title{
Yeast and human TFIIDs are interchangeable for the response to acidic transcriptional activators in vitro
}

\author{
Raymond J. Kelleher III, ${ }^{1}$ Peter M. Flanagan, ${ }^{1}$ Daniel I. Chasman, ${ }^{1}$ Alfred S. Ponticelli, ${ }^{2}$ \\ Kevin Struhl, ${ }^{2}$ and Roger D. Kornberg ${ }^{1,3}$ \\ ${ }^{1}$ Department of Cell Biology, Stanford University School of Medicine, Stanford, California 94305 USA; ${ }^{2}$ Departments of \\ Biological Chemistry and Molecular Pharmacology, Harvard Medical School, Boston, Massachusetts 02115 USA
}

\begin{abstract}
Previous work showed that human TFIID fails to support yeast cell growth, although it is nearly identical to yeast TFIID in a carboxy-terminal region of the molecule that suffices for basal, TATA-element-dependent transcription in vitro. These and other findings raised the possibility that TFIID participates in species-specific interactions, possibly with mediator factors, required for activated transcription. Here, we report that human TFIID and amino-terminally truncated derivatives of yeast TFIID are fully functional in support of both basal transcription and the response to acidic activator proteins in a yeast in vitro transcription system. Conversely, and in contrast to previously published results, yeast TFID supports both basal and activated transcription in reactions reconstituted with human components. This functional interchangeability of yeast and human TFIIDs argues strongly against species specificity with regard to TFIID function in basal transcription and the response to acidic activator proteins. In addition, our results suggest that any intermediary factors between acidic activators and TFIID are conserved from yeast to man.
\end{abstract}

[Key Words: Mediator; TFIID; transcriptional activation; acidic activators; in vitro transcription]

Received October 24, 1991; revised version accepted December 16, 1991.

Transcription factor IID (TFIID), which binds the TATA element of RNA polymerase II promoters, was first defined as a fraction from HeLa cells required for basal transcription (i.e., transcription in the absence of activator proteins). Activity was subsequently shown to reside in a $37-\mathrm{kD}$ polypeptide from human cells (hTFIID), a $27-\mathrm{kD}$ polypeptide from the yeast Saccharomyces cerevisiae (yTFIID), and molecules of comparable size from other organisms (Cavallini et al. 1989; Hahn et al. 1989; Horikoshi et al. 1989; Schmidt et al. 1989; Hoffmann et al. 1990; Kao et al. 1990; Peterson et al. 1990). Much as yTFIID will replace the human protein in basal transcription in the HeLa transcription system, so the HeLa TFIID fraction can be substituted in a yeast system (Buratowski et al. 1988; Flanagan et al. 1990). In keeping with this functional conservation, the sequence of the carboxy-terminal 180 amino acids of TFIID is $81 \%$ identical between yeast and man, and this carboxy-terminal domain, produced either by expression of a deleted gene or by partial proteolysis, is active in basal transcription in the HeLa transcription system (Horikoshi et al. 1990; Peterson et al. 1990; Lieberman et al. 1991).

In contrast with the evolutionary conservation of the carboxy-terminal domain of TFIID, the amino-terminal

${ }^{3}$ Corresponding author. region varies widely in size and sequence, even among closely related organisms. A number of observations have led to the notion that the amino-terminal region is required for transcriptional activation. Deletion of this region from $h$ TFIID eliminates the response to the activator Spl (Peterson et al. 1990). Similarly, yTFIID, with its different amino-terminal region, has been reported to substitute only in basal but not in GAL4-VP16-activated transcription in the HeLa system (Peterson et al. 1990). It was suggested that the amino-terminal region interacts with factors known as mediators or coactivators that are required for activated transcription. Recently, however, the amino-terminal region of yTFIID was shown to be dispensable for viability of yeast, so the amino-terminal region cannot be solely responsible for regulated transcription in this organism (Cormack et al. 1991; Gill and Tjian 1991; Reddy and Hahn 1991; Zhou et al. 1991).

Despite the capacity of the carboxy-terminal domain of yTFIID to perform all essential functions in yeast, hTFIID with a nearly identical carboxy-terminal sequence was inadequate for cell growth (Cormack et al. 1991; Gill and Tiian 1991). This deficiency of hTFIID might be attributed to a failure to support effects of activators in yeast, in keeping with the previous observation that yTFIID is deficient in activated transcription in the HeLa system. Here, we report on studies leading to the opposite conclusion-that human and yeast TFIIDs 
and carboxy-terminal fragments are all functionally equivalent in basal and activated transcription in vitro.

\section{Results}

\section{A TFIID-dependent yeast transcription system responsive to acidic activator proteins}

Yeast extracts have been resolved into five fractions, designated a, b, c, d, and e, which are sufficient along with RNA polymerase II for basal transcription in the absence of activator proteins (Flanagan et al. 1990). Fraction d could be replaced by recombinant yTFIID isolated from Escherichia coli. Upon purification of fraction a, the response of the transcription system to acidic activator proteins was lost, but this response could be restored by the addition of a mediator distinct from the basal transcription factors (Flanagan et al. 1991). As fraction e was further purified, fraction c became dispensable for basal transcription. In this study, a scheme was employed that results in the copurification of mediator and all factors required for basal transcription, with the exception of TFIID, through two chromatographic steps (P.M. Flanagan, R.J. Kelleher, H. Tschochner, M.H. Sayre, and R.D. Kornberg, in prep.). The resulting protein fraction is en- riched $\sim 200$-fold with respect to mediator, RNA polymerase II, and yeast initiation factors $a, b$, and e. Addition of TFIID to this fraction was necessary and sufficient for basal transcription (Fig. 1B). The further addition of fraction $c$ had no stimulatory effect on basal transcription but was important for activation. The complete transcription system supported high levels of activation by GAL4-VP16 and GCN4 with full-length yTFIID (26- and 32-fold, respectively; see Fig. 2), and permitted an analysis of the activities of yTFIID derivatives and hTFIID in basal and activated transcription.

\section{Carboxy-terminal domain of yTFIID is sufficient for basal and activated transcription in a yeast system in vitro}

To assess the importance of the amino-terminal region of yTFIID in basal and activated transcription in vitro, derivatives lacking part or all of this region were expressed in E. coli and purified (Fig. 1A). Derivative yTFIID207C consists of an initiator methionine fused to the 207 carboxy-terminal amino acids, resulting in the deletion of residues 2-33. Derivative yTFIID $179 \mathrm{C}$ consists of an initiator methionine fused to the 179-aminoacid, conserved carboxy-terminal domain, deleting resi-

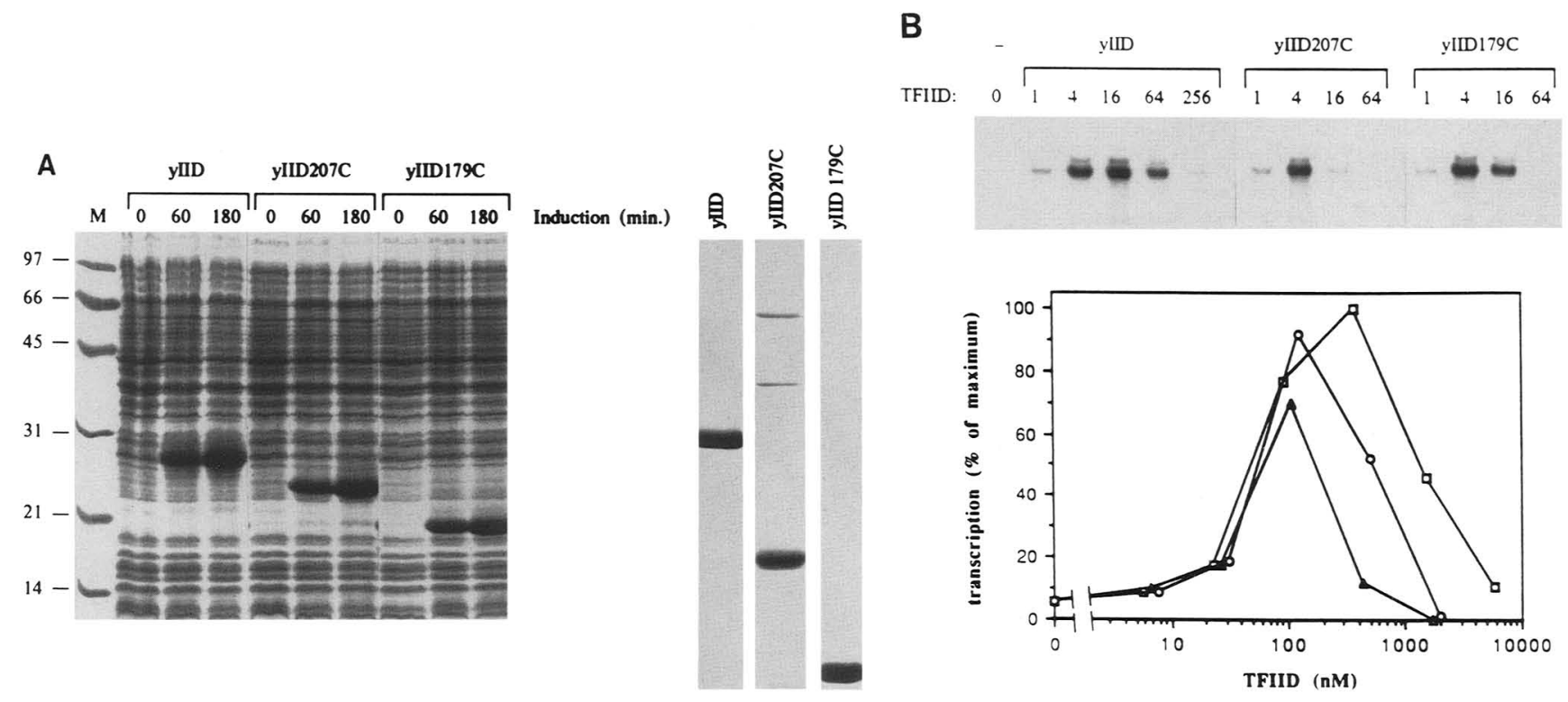

Figure 1. Bacterial expression and activity in basal transcription of yTFID and amino-terminally truncated derivatives. (A) Fulllength yTFIID (yIID) and derivatives consisting of the 207 carboxy-terminal residues (yIID207C) and the 179 carboxy-terminal residues (yIID179C) fused to an initiator methionine, were expressed in E. coli, and purified. (Left) SDS-PAGE analysis of total bacterial protein immediately prior to induction and 1 and $3 \mathrm{hr}$ following induction. (Right) SDS-PAGE analysis of the purified fractions used in transcription assays. $(B)$ Full-length yTFIID and the truncated derivatives were tested for the ability to support basal transcription in a TFIID-dependent reconstituted yeast transcription system. Relative amounts of TFIID assayed are given in multiples of 18.75 ng. Reactions were performed with pGAL4CG - (100 ng), which contains a single GAL4-binding site upstream of the yeast CYC1 promoter fused to a G-less cassette (Lue et al. 1989), in the presence of fraction c, mediator, yeast initiation factors a, b, e, and RNA polymerase II, as described in Results. Radioactivity incorporated into specific transcripts, shown in the autoradiograph, is expressed as a percentage of the maximum (obtained with $300 \mathrm{ng}$ of yTFIID) and plotted as a function of TFIID concentration. ( $\square$ ) yTFIID; ( $\mathbf{\Delta}$ ) yTFIID207C; 101 yTFIID179C. 
A GAL4-VP16 (pmol):
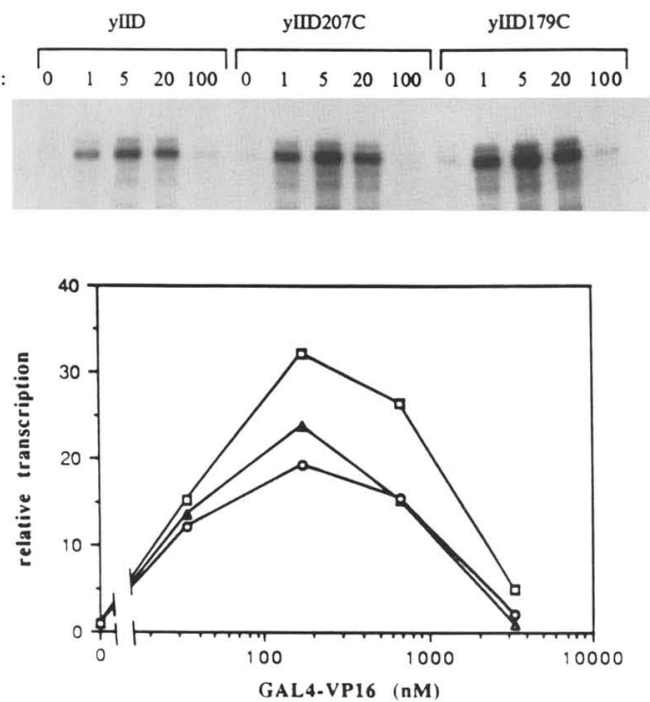

B
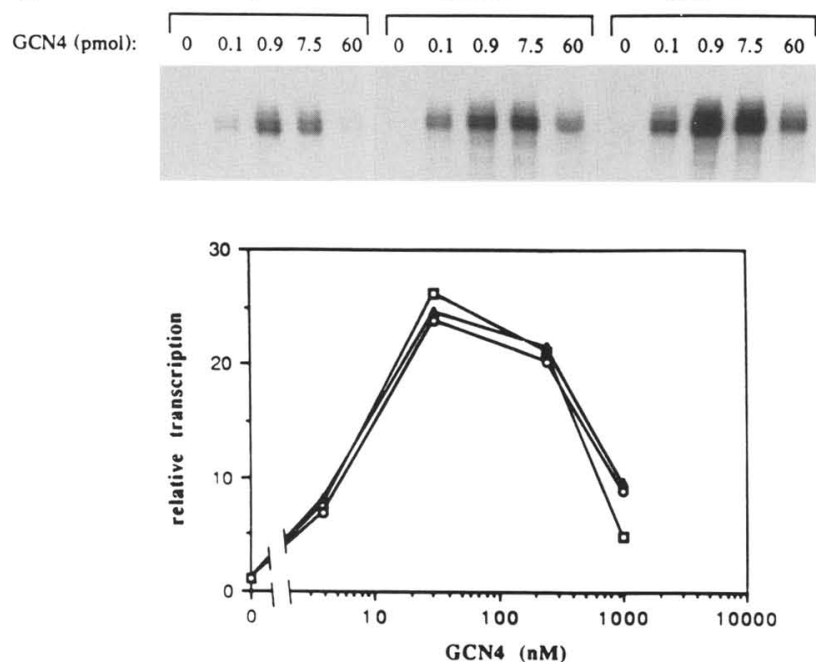

Figure 2. Mediator-dependent response of full-length yTFIID and amino-terminally truncated derivatives to GAL4-VP16 and GCN4. $(A)$ The indicated amounts of GAL4-VP16 were added to transcription reactions, as in Fig. 1B, with the indicated form of TFIID (75 ng) and pGAL4CG - 125 ng). Radioactivity incorporated into spe-

C mediator GAL4-VP16
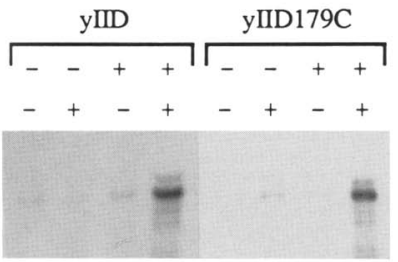

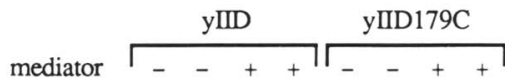

GCN4

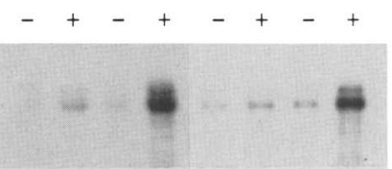
cific transcripts, shown in the autoradiograph, was normalized to that obtained in the absence of activator for each form of TFID and plotted as a function of the GAL4-VP16 concentration (For description of symbols, see Fig. 1B). (D) yTFIID; (A) yTFID207C; $(O)$ yTFIID179C. (B) The indicated amounts of GCN4 were added to transcription reactions, as in Fig. 1B, with the indicated form of TFIID (75 ng) and pSP(GCN4) ${ }^{2} \mathrm{CG}-(25 \mathrm{ng})$, which contains two copies of a GCN4-binding site upstream of the yeast CYC1 promoter fused to a G-less cassette (Flanagan et al. 1990). Radioactivity incorporated into specific transcripts, shown in the autoradiograph, was normalized to that obtained in the absence of activator for each form of TFIID and plotted as a function of GCN4 concentration (see $A$ for description of symbols). (C) Fully reconstituted transcription reactions, containing $100 \mathrm{ng}$ of $\mathrm{pGAL}_{4} \mathrm{CG}^{-}($left $)$or $\mathrm{pSP}(\mathrm{GCN} 4)^{2} \mathrm{CG}^{-}$(right) and the indicated form of TFIID, were performed in the absence $(-)$ or presence $(+)$ of activator protein $(1 \mathrm{pmole})$ and/or highly enriched mediator fraction. All reactions also contained fraction c, RNA polymerase II, and initiation factors $a$, $b$, and $e$ (as described in the Materials and methods). As reported previously (Flanagan et al. 1991), a slight activation of the reconstituted system may be observed in the absence of added mediator, presumably as the result of contamination of fractions supplying yeast general initiation factors with mediator activity. Maximal activation is nonetheless dependent on added mediator.

dues 2-61. Both derivatives exhibited higher DNA-binding activity than full-length yTFIID in gel mobility-shift assays (R.J. Kelleher and W.J. Feaver, unpubl.), as noted previously (Horikoshi et al. 1990).

The activities of the truncated derivatives in support of basal transcription were similar to that of full-length yTFIID in several respects (Fig. 1B). First, the dependence of transcription on low levels of the TFIID molecules was nonlinear, perhaps reflecting the equilibrium binding of the molecules to the TATA element. Second, there was a well-defined optimum, at $\sim 10^{-7} \mathrm{M}$ for the derivatives, and $\sim 4 \times 10^{-7} \mathrm{M}$ for the full-length molecule. The slightly lower optimal concentration for the derivatives might be the result of their somewhat higher affinity for DNA (see above). Finally, all forms of TFIID were very inhibitory at the highest levels tested. In view of the purity of the TFIID preparations and the relatively low concentration at which inhibition was observed $\left(10^{-6}\right.$ $\left.10^{-7} \mathrm{M}\right)$, this effect may be specific and not attributable to a contaminant.

The two truncated derivatives of TFIID were indistinguishable from the full-length molecule in support of activation by GCN4 and only slightly less effective than the full-length molecule in activation by GAL4-VP16 (Fig. 2A,B). The small difference in behavior might be explained by the presence of two activator-binding sites in the GCN4-responsive template, as opposed to one site in the GAL4 template, or the amino terminus might make some minor contribution to the response to GAL4-VP16. The overall levels of transcription were higher with the truncated TFIID derivatives /see autoradiographs, Fig. 2A,B, top), probably reflecting the increased DNA-binding activities of the derivatives. No effect of the activators was seen in the absence of added TFIID (data not shown), demonstrating that activation was entirely the result of the added form of TFIID and 
not any of the full-length molecule contaminating the fractions used to reconstitute transcription.

Although the carboxy-terminal domain of yTFID supported activation by GAL4-VP16 and GCN4, ruling out a requirement for the amino-terminal region, the possibility remained that the amino-terminal region functions as a negative element, as indicated in other circumstances (Horikoshi et al. 1990, and see above). According to this hypothesis, mediator would be required to relieve the inhibitory effect of the amino-terminal region, whereas activators would function through direct contact with the carboxy-terminal domain of TFIID or another general initiation factor. If this were the case, mediator would only be required for activation with fulllength TFIID but not with truncated derivatives. However, we found that mediator was essential for activation by GAL4-VP16 and GCN4 with both full-length and amino-terminally truncated TFIID in a fully reconstituted yeast transcription system (Fig. 2C). Taken together, our results indicate that the amino-terminal region of yTFIID does not play a significant role in the response of the general transcription machinery to these acidic activators and that if YTFIID does participate in regulatory interactions with acidic activators or the mediator, these interactions are restricted to the carboxyterminal domain.

\section{hTFIID and yTFIIDs are functionally interchangeable in transcription in vitro}

The capacity of the carboxy-terminal domain of yTFIID to support both basal and activated transcription in the yeast system and the sequence conservation of this domain between yeast and man lead one to expect that hTFIID would be fully functional in yeast as well. And yet, as mentioned above, hTFIID fails to support yeast cell growth. To determine whether this failure of hTFID reflects a general transcriptional defect, we investigated the activity of hTFIID in the yeast system in vitro.

Recombinant hTFIID supported basal transcription from both the yeast $C Y C 1$ promoter and the adenoviral major late promoter (Fig. 3A). As described above for yTFIID and amino-terminally deleted derivatives, the concentration dependence of transcription at low levels of hTFIID was nonlinear. Transcription was maximal at $\sim 10^{-7} \mathrm{M}$, and higher concentrations were inhibitory. The maximal level of basal transcription obtained with hTFIID from the CYCl and adenoviral major late promoters was $\sim 45 \%$ and $70 \%$, respectively, of that obtained with full-length yTFIID from the same promoters. Recombinant hTFIID also supported activation by both GAL4-VP16 and GCN4 over the same range of activator concentration as observed with yTFIID and derivatives (Fig. 3B,C). We can therefore rule out a general defect of hTFIID in basal transcription or in the response to acidic activators of either mammalian or yeast origin in the yeast system.

Because hTFIID supports the effect of acidic activators in the yeast transcription system, yTFIID ought to do the same in the HeLa transcription system. To investigate this point, TFIID activity in a HeLa nuclear extract was inactivated by heat treatment (Nakajima et al. 1988). Addition of yTFIID to the heated extract restored both basal and activated transcription to levels similar to those obtained with unheated extract (Fig. 4). The amino-terminally truncated yTFIID derivatives yTFIID179C and yTFIID207C also fully restored the response of the heated extract to GAL4-VP16 (data not shown), further emphasizing the sufficiency of the conserved carboxyterminal domain for this response. Thus, human and yeast TFIIDs are interchangeable between human and yeast systems with regard to both basal transcription and effects of acidic activators.

\section{Discussion}

The findings reported here resolve two paradoxes regarding the structure and function of TFIID. First, it was noted previously that the carboxy-terminal domain of TFIID, $>80 \%$ conserved between yeast and man, is sufficient for basal transcription in the HeLa system, yet hTFIID is inadequate for yeast cell growth (Horikoshi et al. 1990; Peterson et al. 1990; Cormack et al. 1991; Gill and Tiian 1991). The present results demonstrate that hTFIID is fully functional in a yeast transcription system in vitro, so the insufficiency in vivo cannot be due to a general defect in support of yeast RNA polymerase II transcription. A number of alternatives may be considered: hTFIID may fail to support RNA polymerase II transcription of one or a small number of essential genes; it may not respond to other types of activation domains (i.e., nonacidic); it may fail to act as required at RNA polymerase III promoters (Margottin et al. 1991; Simmen et al. 1991); or it may fail to fulfill an essential role unrelated to transcription.

A second paradox arose from the previous proposal (Peterson et al. 1990) that the amino-terminal region of TFIID, although highly divergent across species, is involved in the response to a "universal" activator, GAL4VP16, which stimulates transcription of responsive genes in both yeast and HeLa transcription systems (Sadowski et al. 1988; Chasman et al. 1989; Kelleher et al. 1990). This proposal was based on the failure of yTFIID to support activation by GAL4-VP16 in a HeLa transcription system (Peterson et al. 1990). We have now shown that on the contrary, yTFIID does support a response to GAL4-VP16 in the HeLa system comparable to that observed with the endogenous hTFIID; and although the reason for the discrepancy is unclear, our positive result seems more likely to be correct than the previous negative one. Moreover, our findings are in accord with the recent demonstrations that YTFIID supports a GAL4-VP16 effect in the HeLa system in the context of a chromatin template (Workman et al. 1991) and that yTFIID can partially restore the GAL4-VP16 response of a HeLa nuclear extract subjected to an appropriate heat treatment (White et al. 1991). Our findings do not bear on a possible role of the amino-terminal region of hTFID in the response to other classes of activator, such as Spl (Peterson et al. 1990). 
The growth of yeast cells bearing only the carboxyterminal domain of yTFIID has been variously reported to be indistinguishable from that of wild-type yeast (Cormack et al. 1991; Reddy and Hahn 1991) or to be much impaired (Gill and Tjian 1991; Zhou et al. 1991). In the latter case, the poor growth phenotype was attributed to the deletion of a small amino-terminal region of yTFIID between residues 48 and 57 (Zhou et al. 1991). In the present study, yTFIID derivatives both containing (yTFIID207C) and lacking (yTFIID179C) these residues exhibited comparable activities to the full-length molecule both in basal transcription and in the response to acidic activators, indicating that the deletion of this region does not impose any general transcriptional defect. These findings from transcription in vitro are in agreement with a report that inducible transcription from the GAL1 and CYC1 promoters in vivo is unaffected by deletion of the amino-terminal region of yTFIID (Reddy and Hahn 1991|. In addition, yeast strains bearing only the carboxy-terminal domain of yTFIID are viable under selective conditions requiring activation by GCN4,
ACE1, and GAL4 proteins (Cormack et al. 1991). Although we did observe minor differences in optimal concentrations for basal transcription among yTFIID and the amino-terminally truncated derivatives, these differences are unlikely to account for the importance of the amino-terminal region in vivo in some studies, because the growth defect was observed over a wide range of expression levels of the carboxy-terminal domain (Zhou et al. 1991|. Rather, deletion of the amino-terminal region may affect the expression of one or a small number of essential genes in a manner analogous to that noted with reporter genes bearing upstream elements of the CUP1 or HSP promoters (Zhou et al. 1991).

The inhibitory effect of TFIID at high concentrations found here resembles that observed previously in vitro (Kelleher et al. 1990) and in vivo (Gill and Ptashne 1988; Triezenberg et al. 1988; Meyer et al. 1989; Kelleher et al. 1990; Martin et al. 1990) at high concentrations of activator proteins, and it may have a similar explanation: TFIID may sequester another general initiation factor with which it interacts in the transcription process. Ex-
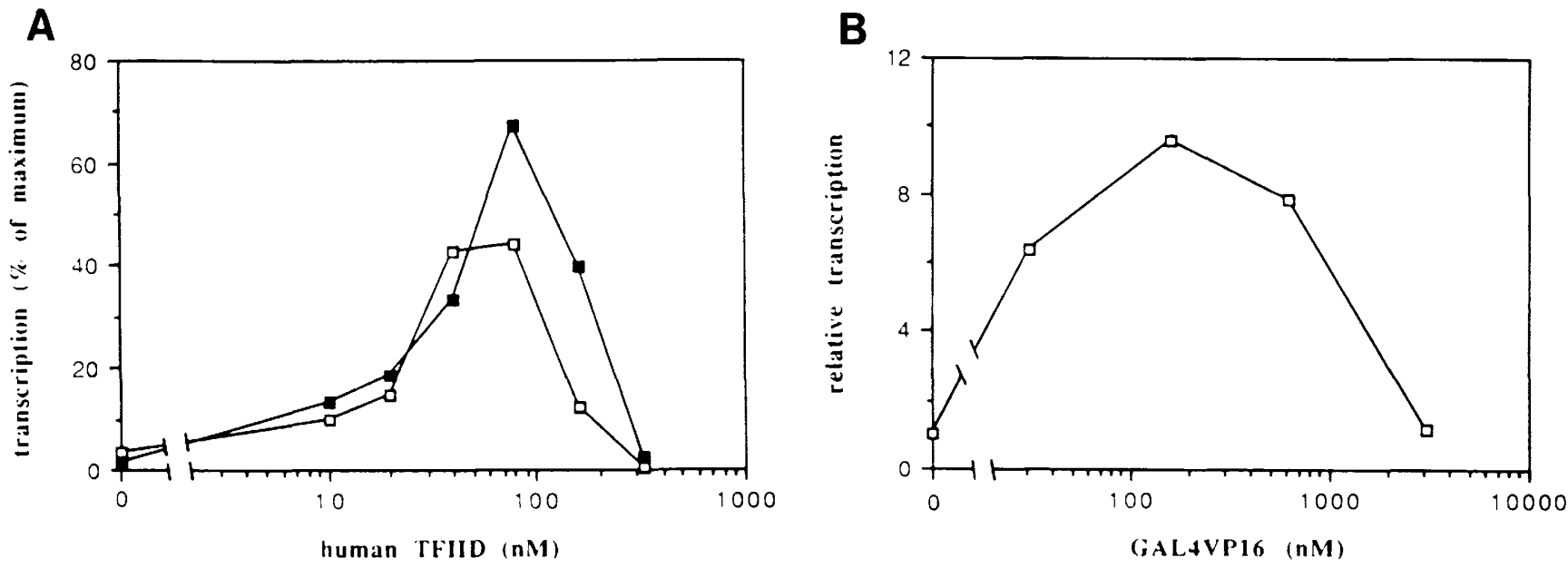

Figure 3. hTFIID supports basal transcription and stimulation by acidic activators in the yeast transcription system. $(A \mid$ Increasing amounts of hTFID were tested for the ability to support basal transcription from the yeast $C Y C 1$ and adenoviral major late promoters in the TFIID-dependent reconstituted yeast transcription system. The indicated amounts of purified recombinant hTFIID were added to transcription reactions, as in Fig. 1B, containing $100 \mathrm{ng}$ of either pGAL4CG $-(\square$; $\mathrm{CYCl})$ or $\mathrm{pML}\left(\mathrm{C}_{2} \mathrm{AT}\right)_{19}(\mathbf{\square}$; adenoviral major late promoter; Sawadogo and Roeder 1985a). Basal transcription from each promoter is expressed as a percentage of the maximum transcription obtained with yTFIID and the same promoter under identical conditions and plotted as a function of hTFID concentration. $(B)$ GAL4-VP16 was added in increasing amounts to transcription reactions, as in Fig. 1B, containing pGAL4CG - (30 ng) and hTFIID (100 ng). Radioactivity incorporated into specific transcripts was normalized to that obtained in the absence of activator and plotted as a function of

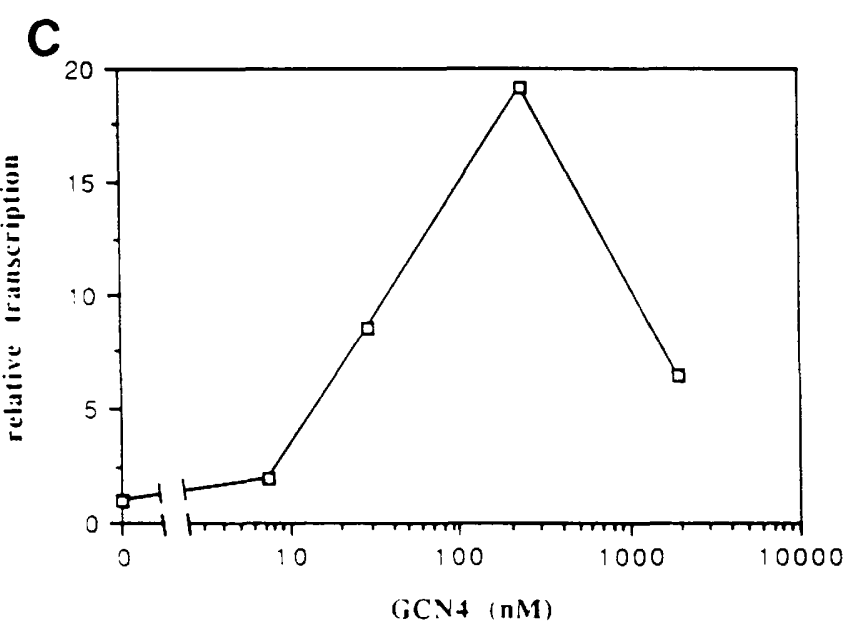
GAL4-VP16 concentration. $(C)$ GCN4 was added in increasing amounts to transcription reactions, as in Fig. $1 \mathrm{~B}$, containing pSP(GCN4) ${ }^{2} \mathrm{CG}-(30 \mathrm{ng})$ and hTFID (100 ng). Radioactivity incorporated into specific transcripts was normalized to that obtained in the absence of activator and plotted as a function of GCN4 concentration. 


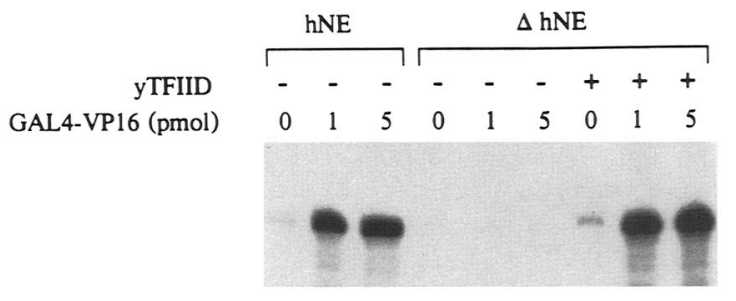

Figure 4. yTFIID supports a GAL4-VP16 response in a HeLa transcription system. The indicated amounts of GAL4-VP16 were added to transcription reactions containing pGAL4MLG(100 ng), with a single GAL4-binding site upstream of the adenovirus major late promoter fused to a G-less cassette (Lue et al. 1989), and untreated HeLa nuclear extract (hNE), heat-treated HeLa nuclear extract, or heat-treated HeLa nuclear extract $(\triangle \mathrm{hNE})$ supplemented with recombinant yTFIID.

pression of TFIID at high levels in vivo impairs cell growth (Reddy and Hahn 1991), suggesting that the effect we find in vitro may indeed be functionally significant. The possibility remains, however, that the inhibitory effect has a trivial explanation. While it was probably not caused by a contaminant, for the reasons mentioned above, it could have been the result of nonspecific interaction of TFIID with the template DNA or self-aggregation of the protein.

Stimulation of yeast RNA polymerase II transcription by the acidic activators GAL4-VP16 and GCN4 is dependent on a mediator distinct from the general initiation factors (Kelleher et al. 1990; Flanagan et al. 1991). The action of such acidic activator proteins and mediator may involve contact(s) with general initiation factors, and TFIID has been implicated as a target of such interactions (Sawadogo and Roeder 1985b; Horikoshi et al. 1988; Peterson et al. 1990; Stringer et al. 1990; Ingles et al. 1991). The ability of the carboxy-terminal domain of yTFIID to support activation by GAL4-VP16 and GCN4 in a mediator-dependent fashion eliminates the aminoterminal region as a potential target of interactions with either acidic activation domains or the mediator. Likewise, the capacity of YTFIID to support effects of GAL4VP16 in the HeLa transcription system shows that the amino-terminal region of the human protein is dispensable for activation and further suggests that any interactions of acidic activators or a putative HeLa mediator with TFIID are not species-specific. The lack of a requirement for the divergent amino-terminal region and the interchangeability of the conserved carboxy-terminal domain of TFIID between yeast and human systems described here could account for the evolutionary conservation of the response to acidic activators. If the conserved carboxy-terminal domain of TFIID is a target of mediator, our results suggest further that the mediator itself is conserved between yeast and man.

\section{Materials and methods}

Preparation of TFIID

The full-length yeast TFIID gene was modified to create an NdeI site at the ATG by replacement of sequences between the start site and the DraI site at amino acid 6 with a synthetic oligonucleotide. Amino-terminally truncated derivatives were generated by the polymerase chain reaction with upstream primers containing NdeI sites followed by internal TFIID-coding sequences. The complete coding sequence and the two PCR-derived deletions were then cloned as NdeI-BamHI fragments into the T7 polymerase expression vector pET1 $1 \mathrm{a}$ and expressed in E. coli strain BL21(DE3) as described (Studier and Moffat 1986). The amino acid sequence of the yTFIDI79C derivative was confirmed by determination of the nucleotide sequence. The expressed yTFIID and yTFIID179C polypeptides were purified to homogeneity by a chromatographic scheme to be described elsewhere (D.I. Chasman et al., in prep.). The yTFIID207C derivative was purified to near homogeneity by chromatography over DE52 and heparin-Sepharose essentially as described (Hoey et al. 1990). hTFID was expressed in BL21(DE3) from the T7 polymerase expression vector pET3a containing a full-length HeLa TFIID cDNA (generous gift of A. Hoffmann and R. Roeder). The bacterial lysate in buffer A 300 mM HEPES-KOH ( $\mathrm{pH} 7.6), 10 \%$ glycerol, $1 \mathrm{~mm}$ EDTA, $0.5 \mathrm{~mm}$ DTT, $1 \mathrm{mM}$ PMSF; concentration of $\mathrm{KCl}$ in $\mathrm{mM}$ in parentheses) containing $0.2 \mathrm{M} \mathrm{KCl}$ was applied to a DE52 column equilibrated in the same buffer. The flowthrough was diluted to 100 $\mathrm{mM} \mathrm{KCl}$ and applied to a heparin-Sepharose column equilibrated in buffer $\mathrm{A}(100)$. The column was successively eluted with buffer A containing $0.2 \mathrm{M}, 0.6 \mathrm{M}$, and $1 \mathrm{M} \mathrm{KCl}$. The $0.6 \mathrm{M}$ eluate was dialyzed against buffer A $(90)$ and applied to a Q-Sepharose column equilibrated in buffer A (90). The flow through from Q-Sepharose was then applied to a SP-5PW column equilibrated in buffer $\mathrm{A}(90)$. The column was washed with buffer $\mathrm{A}(200)$ and developed with a linear gradient from $0.2-1 \mathrm{M}$ $\mathrm{KCl}$. The peak of hTFIID eluted at $\sim 0.38 \mathrm{M} \mathrm{KCl}$ and was estimated to be $50 \%$ pure by SDS-PAGE.

\section{In vitro transcription}

GAL4-VP16 protein (Chasman et al. 1989) and GCN4 protein (generous gifts of C. Ampe and T. Steitz) were expressed in $E$. coli and purified. Fraction c was prepared from yeast nuclear extracts as described previously (Flanagan et al. 1990). For all transcription reactions except those in Figure $2 \mathrm{C}$, yeast initiation factors $a, b, e$, and RNA polymerase II, in addition to mediator, were supplied by a single fraction enriched $\sim 200$-fold by chromatography of yeast whole-cell extracts on Bio-Rex70 (BioRad) and DE52 (Whatman) by methods described elsewhere (P.M. Flanagan, H. Tschochner, R.J. Kelleher, M.H. Sayre, and R.D. Kornberg, in prep.). Basal and activated transcription reactions were reconstituted with $8-10 \mu \mathrm{g}$ of this fraction, $3 \mu \mathrm{g}$ of fraction $c$, the indicated amount of TFIID, and activator protein, where appropriate. For the transcription reactions in Figure 2C, yeast initiation factors $a, b$, and RNA polymerase II were prepared as described (Edwards et al. 1990; Feaver et al. 1991; Flanagan et al. 1991). Mediator, prepared as described (Flanagan et al. 1991), was subjected further to high-performance liquid chromatography (HPLC) on a Bio-Gel heparin-5PW column. Yeast initiation factor e was obtained from further chromatography of the single enriched fraction, described above, on a SP5PW column (H. Tschochner, unpubl.). HeLa nuclear extract was heated for $15 \mathrm{~min}$ at $47^{\circ} \mathrm{C}$ to inactivate endogenous TFIID activity (Nakajima et al. 1988). Recombinant yTFIID employed in the HeLa transcription assays was prepared as described (Flanagan et al. 1990). Yeast and HeLa transcription reactions were performed and analyzed as described previously for yeast nuclear extracts (Lue et al. 1989; Kelleher et al. 1990). 


\section{Acknowledgments}

We thank C. Ampe and T. Steitz for the generous provision of GCN4 protein, A. Hoffmann and R. Roeder for the hTFIID expression plasmid, and F. Pugh, R. Tjian, E. Sinn, and R. Roeder for HeLa nuclear extract. R.J.K. was supported by the Medical Scientist Training Program [National Institutes of Health (NIH) grant GM-07365]. A.S.P. was supported by a Helen Hay Whitney postdoctoral fellowship. This work was supported by research grants to R.D.K. from the NIH (GM-36659, AI-21144) and to K.S. from the NIH (GM-30186) and the Lucille Markey Trust.

The publication costs of this article were defrayed in part by payment of page charges. This article must therefore be hereby marked "advertisement" in accordance with 18 USC section 1734 solely to indicate this fact.

\section{References}

Buratowski, S., S. Hahn, P.A. Sharp, and L. Guarente. 1988. Function of a yeast TATA element binding protein in a mammalian transcription system. Nature 334: 37-42.

Cavallini, B., I. Faus, H. Matthes, J.-M. Chipoulet, B. Winsor, J.-M. Egly, and P. Chambon. 1989. Cloning of the gene encoding the yeast protein BTFl, which can substitute for the human TATA box-binding factor. Proc. Natl. Acad. Sci. 89: 9803-9807.

Chasman, D.I., J. Leatherwood, M. Carey, M. Ptashne, and R.D. Kornberg. 1989. Activation of yeast RNA polymerase II transcription by herpes VP16 and GAL4 derivatives in vitro. Mol. Cell. Biol. 9: 4746-4749.

Cormack, B.P., M. Strubin, A.S. Ponticelli, and K. Struhl. 1991. Functional differences between yeast and human TFIID are localized to the highly conserved region. Cell 65: 341-348.

Edwards, A.M., S.A. Darst, W.J. Feaver, N.E. Thompson, R.R. Burgess, and R.D. Kornberg. 1990. Purification and lipid layer crystallization of yeast RNA polymerase II. Proc. Natl. Acad. Sci. 87: 2122-2126.

Feaver, W.J., O. Gileadi, and R.D. Kornberg. 1991. Purification and characterization of yeast RNA polymerase II transcription factor b. I. Biol. Chem. 266: 19000-19005.

Flanagan, P.M., R.J. Kelleher III, W.J. Feaver, N.F. Lue, J.W. LaPointe, and R.D. Kornberg. 1990. Resolution of factors required for the initiation of transcription by yeast RNA polymerase II. J. Biol. Chem. 265: 11105-11107.

Flanagan, P.M., R.J. Kelleher III, M.H. Sayre, H. Tschochner, and R.D. Kornberg. 1991. A mediator required for activation of RNA polymerase II transcription in vitro. Nature 350: 436438.

Gill, G. and M. Ptashne. 1988. Negative effect of the transcriptional activator GAL4. Nature 334: 721-724.

Gill, G. and R. Tiian. 1991. A highly conserved domain of TFID displays species specificity in vivo. Cell 65: 333-340.

Hahn, S., S. Buratowski, P.A. Sharp, and L. Guarente. 1989. Isolation of the gene encoding the yeast TATA binding protein TFIID: A gene identical to the SPT15 suppressor of Ty element insertions. Cell 58: 1173-1181.

Hoey, T., B.D. Dynlacht, M.G. Peterson, B.F. Pugh, and R. Tjian. 1990. Isolation and characterization of the Drosophila gene encoding the TATA box binding protein, TFIID. Cell 61: 1179-1186.

Hoffmann, A., E. Sinn, Y.T.J. Wang, A. Roy, M. Horikoshi, and R.G. Roeder. 1990. Highly conserved core domain and unique $\mathrm{N}$-terminal region with presumptive regulatory motifs in a human TATA factor (TFIID). Nature 346: 387-390.

Horikoshi, M., M.F. Carey, H. Kakidani, and R.G. Roeder. 1988. Mechanism of activation of a yeast activator: Direct effect of
GAL4 derivatives on mammalian TFIID-promoter interactions. Cell 54: 665-669.

Horikoshi, M., C.K. Wang, H. Fujii, J.A. Cromlish, P.A. Weil, and R.G. Roeder. 1989. Cloning and structure of a yeast gene encoding a general transcription initiation factor TFIID that binds to the TATA box. Nature 341: 299-303.

Horikoshi, M., T. Yamamoto, Y. Ohkuma, P.A. Weil, and R.G. Roeder. 1990. Analysis of structure-function relationships of yeast TATA box binding factor TFIID. Cell 61: 1171-1178.

Ingles, C.J., M. Shales, W.D. Cress, S.J. Triezenberg, and J. Greenblatt. 1991. Reduced binding of TFIID to transcriptionally compromised mutants of VP16. Nature 351: 588-590.

Kao, C.C., P.M. Lieberman, M.C. Schmidt, Q. Zhou, R. Pei, and A.J. Berk. 1990. Cloning of the gene encoding the human TATA binding factor: Expression and transcriptionally active TFIID protein. Science 248: 1646-1650.

Kelleher, R.J. III, P.M. Flanagan, and R.D. Kornberg. 1990. A novel mediator between activator proteins and the RNA polymerase II transcription apparatus. Cell 61: 1209-1215.

Lieberman, P.M., M.C. Schmidt, C.C. Kao, and A.J. Berk. 1991. Two distinct domains in the yeast transcription factor IID and evidence for a TATA box-induced conformational change. Mol. Cell. Biol. 11: 63-74.

Lue, N.F., P.M. Flanagan, K. Sugimoto, and R.D. Kornberg. 1989. Initiation by yeast RNA polymerase II at the adenoviral major late promoter in vitro. Science 246: 661-664.

Margottin, F., G. Dujardin, M. Gerard, J.-M. Egly, J. Huet, and A. Sentenac. 1991. Participation of the TATA factor in transcription of the yeast U6 gene by RNA polymerase C. Science 251: 424426.

Martin, K.J., J.W. Lillic, and M.R. Green. 1990. Evidence for interaction of different eukaryotic transcriptional activators with distinct cellular targets. Nature 346: 147-152.

Meyer, M.-E., H. Gronemeyer, B. Turcotte, M.-T. Bocquel, D. Tassett, and P. Chambon. 1989. Steroid hormone receptors compete for factors that mediate their enhancer function. Cell 57: 433-442.

Nakajima, N., M. Horikoshi, and R.G. Roeder. 1988. Factors involved in specific transcription by mammalian RNA polymerase II: Purification, genetic specificity, and TATA boxpromoter interactions of TFIID. Mol. Cell. Biol. 8: 40284040.

Peterson, M.G., N. Tanese, B.F. Pugh, and R. Tjian. 1990. Functional domains and upstream activation properties of cloned human TATA binding proteins. Science 248: 1625-1630.

Reddy, P. and S. Hahn. 1991. Dominant negative mutations in yeast TFIID define a bipartite DNA-binding region. Cell 65: 349-357.

Sadowski, I., J. Ma, S. Triezenberg, and M. Ptashne. 1988. GAL4VP16 is an unusually potent transcriptional activator. $\mathrm{Na}$ ture 335: 563-564.

Sawadogo, M. and R.G. Roeder. 1985a. Factors involved in specific transcription by hunam RNA polymerase II: Analysis by a rapid and quantitative in vitro assay. Proc. Natl. Acad. Sci. 82: 4394-4398.

- 1985b. Interaction of a gene specific transcription factor with the adenovirus major late promoter upstream of the TATA box region. Cell 43: 165-175.

Schmidt, M.C., C.C. Kao, R. Pei, and A.J. Berk. 1989. Yeast TATA-box transcription factor gene. Proc. Natl. Acad. Sci. 86: 7785-7789.

Simmen, K.A., J. Bernues, H.D. Parry, H.G. Stunnenberg, A. Berkenstam, B. Cavallini, J.-M. Egly, and I.W. Mattaj. 1991. TFIID is required for in vitro transcription of the human U6 gene by RNA polymerase III. EMBO I. 10: 1853-1862.

Stringer, K.F., C.J. Ingles, and J. Greenblatt. 1990. Direct and 
selective binding of an acidic transcriptional activation domain to the TATA-box factor TFIID. Nature 345: 783-786.

Studier, F.W. and B.A. Moffat. 1986. Use of bacteriophage T7 RNA polymerase to direct high level expression of cloned genes. I. Mol. Biol. 189: 113-130.

Triezenberg, S.J., R.C. Kingsbury, and S.L. McKnight. 1988. Functional dissection of VP16, the trans-activator of herpes simplex virus immediate early gene expression. Genes \& Dev. 2: 718-729.

White, J.H., C. Brou, J. Wu, N. Burton, J.-M. Egly, and P. Chambon. 1991. Evidence for a factor required for transcriptional stimulation by the chimeric acidic activator GAL4-VP16 in HeLa cell extracts. Proc. Natl. Acad. Sci. 88: 7674-7678.

Workman, J.L., I.C.A. Taylor, and R.E. Kingston. 1991. Activation domains of stably bound GAL4 derivatives alleviate repression of promoters by nucleosomes. Cell 64: 533-544.

Zhou, Q., M.C. Schmidt, and A.J. Berk. 1991. Requirement for acidic amino acid residues immediately $\mathrm{N}$-terminal to the conserved domain of Saccharomyces cerevisiae TFIID. $E M B O$ /. 10: 1843-1852. 


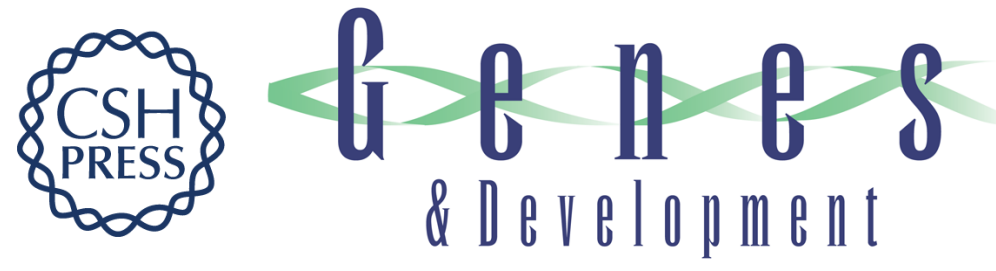

\section{Yeast and human TFIIDs are interchangeable for the response to acidic transcriptional activators in vitro.}

R J Kelleher, P M Flanagan, D I Chasman, et al.

Genes Dev. 1992, 6:

Access the most recent version at doi:10.1101/gad.6.2.296

References This article cites 38 articles, 14 of which can be accessed free at:

http://genesdev.cshlp.org/content/6/2/296.full.html\#ref-list-1

License

Email Alerting

Service

Receive free email alerts when new articles cite this article - sign up in the box at the top right corner of the article or click here.

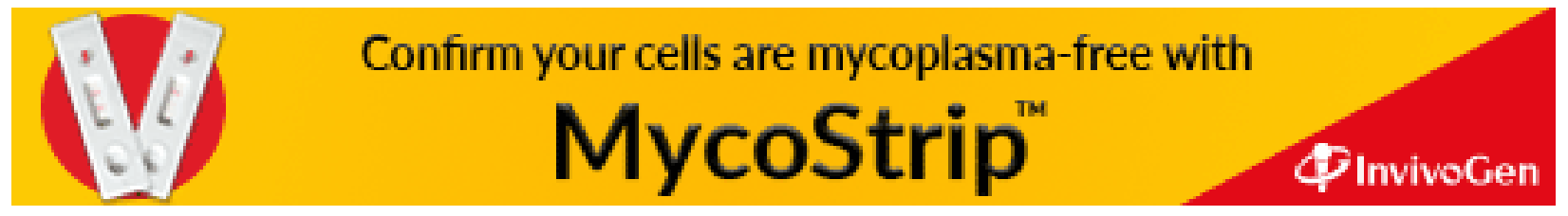

